S. HAMADA

KODAI MATH. J

6 (1983), $47-50$

\title{
NORM THEOREM ON SPLITTING FIELDS OF SOME BINOMIAL POLYNOMIALS
}

\author{
By Suguru HAMADA
}

Let $K$ be a finite algebraic number field and let $M / K$ be a finite Galois extension. Let Knot $(M / K)$ be the factor group $\left\{a \in K^{\times}, a\right.$ is a local norm everywhere $\} /\left\{a \in K^{\times}, a\right.$ is a global norm $\}$. Hasse's norm theorem asserts that if $M / K$ is a cyclic extension then $\operatorname{Knot}(M / K)=1$. H. HASSE ([4]) showed that the norm theorem not always holds for arbitrary abelian extension by giving a counter example: $M=\boldsymbol{Q}(\sqrt{ }-39, \sqrt{-3})$ and $K=\boldsymbol{Q}$, where $\boldsymbol{Q}$ is the field of rational numbers.

And related theories are in [1], [2], [3], [6], and [7]. In this paper we prove the following:

THEOREM. Let $p$ be an odd prime number, $\zeta$ a primitıve $p^{r}$-th root of unity $(r \geqq 1), K$ a finite algebraic number field, $L=K(\zeta)$ and $M=L\left(a^{1 / p^{r}}\right)(a \in K)$.

If $f(X)=X^{p^{r}}-a$ is irreducible in $L[X]$ then $K$ not $(M / K)=1$. When $\sqrt{ }-1 \in K$ the same assertion holds also for $p=2$.

In Remark, by examples, we shall show that in Theorem if we replace $p^{r}$ by a number which is not a power of an odd prime number or by $2^{r}(r \geqq 2$ and $\sqrt{-1} \notin K)$ then the conclusion is not always valid.

In $\S 1$, we shall prove Theorem and Remark by determing $\operatorname{Knot}(M / K)$ explicitely by the following Lemma:

LemMa. Let $l, n$ be positive integers and let $G$ be a group of order $\ln$ generated by two elements $\sigma, \tau$ whose fundamental relations are $\sigma^{l}=\tau^{n}=1, \tau \sigma \tau^{-1}=\sigma^{m}$ $\left(1 \leqq m<l\right.$ and $m^{n}-1$ is a multipler of $\left.l\right)$. Then $H^{3}(G, Z) \approx Z / d Z$ where $d=(1+$ $\left.m+\cdots+m^{n-1}, l,\left(m^{n}-1\right) / l, m-1\right)$ and $Z$ is the ring of rational integers on which $G$ operates trivially. in $[4]$.

In $\S 2$, we shall give a proof of the Lemma as a corollary of a proposition

\section{$\S 1$. Proofs of Theorem and Remark.}

In the following the notations are same as those in our Theorem. Let $G=$

Received January 13, 1982 
$\operatorname{Gal}(M / K)$ be the Galois group of $M / K, M^{\times}$the multiplicative group of $M, J_{M}$ the idèle group of $M$, and $C_{M}$ the idèle class group of $M$.

Then the exact sequence

$$
1 \longrightarrow M^{\times} \longrightarrow J_{M} \longrightarrow C_{M} \longrightarrow 1
$$

gives an exact sequence

$$
\cdots \longrightarrow H^{-1}\left(G, C_{M}\right) \longrightarrow H^{\circ}\left(G, M^{\times}\right) \longrightarrow H^{\circ}\left(G, J_{M}\right) \longrightarrow H^{\circ}\left(G, C_{M}\right) \longrightarrow \cdots .
$$

By Tate's Theorem, we have $H^{-1}\left(G, C_{M}\right) \approx H^{-3}(G, Z)$. In the following, by Lemma we show that $H^{-3}(G, Z)=0$ then we have an exact sequence $1 \rightarrow H^{\circ}\left(G, M^{\times}\right)$ $\rightarrow H^{\circ}\left(G, J_{M}\right)$.

Therefore, the canonical map $K^{\times} / N_{M / K} M^{\times} \rightarrow J_{K} / N_{M / K} J_{M}$ is injective and we have Theorem. Now we show that $H^{3}(G, Z)=0$ by Lemma then $H^{-3}(G, Z)=0$ follows because in general $H^{-3}(G, Z) \approx H^{3}(G, Z)$.

First let $p \neq 2,[L: K]=n, \theta=a^{1 / p^{r}}$ and $\rho$ a rational integer such that $\rho$ $\bmod p^{r}$ generates the units group of $Z / p^{r} Z$. By assumption, $M / L$ is a cyclic Kummer extension of degree $p^{r}$ and $L / K$ is also a cyclic extension of degree $n$. Let $\sigma, \tau$ be the elements of $G$ such that $\sigma(\theta)=\theta \zeta, \sigma(\zeta)=\zeta ; \tau(\theta)=\theta, \tau(\zeta)=\zeta^{m}$, where $m \equiv \rho^{\varphi\left(p^{r}\right) / n} \bmod p^{r}\left(\varphi\right.$ is Euler's function and $\left.1 \leqq m<p^{r}\right)$.

Then $G=\langle\sigma, \tau\rangle, \sigma^{p^{r}}=\tau^{n}=1, \tau \sigma \tau^{-1}=\sigma^{m}$ and $G$ is a group of the type in Lemma. Therefore, we have $H^{3}(G, Z)=Z / d Z$ where $d=\left(1+m+\cdots+m^{n-1}, p^{r}\right.$, $\left.\left(m^{n}-1\right) / p^{r}, m-1\right)$. We show that $d=1$.

Now, $d \neq 1$ if and only if $m \equiv 1 \bmod p, n \equiv 0 \bmod p$ and $\left(m^{n}-1\right) / p^{r} \equiv 0 \bmod p$. While if $n \equiv 0 \bmod p$, we have $m^{n} \equiv \rho^{\varphi\left(p^{r}\right)} \bmod p^{r+1}$ and $\rho^{\varphi\left(p^{r}\right)} \equiv 1 \bmod p^{r+1}$, because in fact $n$ is a divisor of $\varphi\left(p^{r}\right)$ and $n \equiv 0 \bmod p$ implies $r \geqq 2$. Therefore we have $\left(m^{n}-1\right) / p^{r} \not \equiv 0 \bmod p$ and $d=1$.

Next let $p=2, \sqrt{-1} \in K$ and $[L: K]=n$. If $r \leqq 2$ we have the result immediately, so let $r \geqq 3$. Since $\sqrt{-1} \in K, \operatorname{Gal}(L / K)$ is also a cyclic group generated by $\tau_{0}$ such that $\tau_{0}(\zeta)=\zeta^{m}$ where $m \equiv 5^{2^{r-1 / n}} \bmod 2^{r}$ and $1 \leqq m<2^{r}$.

And $G=\langle\sigma, \tau\rangle\left(\sigma(\theta)=\theta \zeta, \sigma(\zeta)=\zeta ; \tau(\theta)=\theta, \tau(\zeta)=\zeta^{m}\right), \sigma^{2^{r}}=\tau^{n}=1$ and $\tau \sigma \tau^{-1}=$ $\sigma^{m}$. Now if $n \equiv 0 \bmod 2$ we have $m^{n} \equiv 5^{2^{r-2}} \bmod 2^{r+1}$, and $5^{2^{r-2}} \not \equiv 1 \bmod 2^{r+1}$. Therefore $H^{3}(G, Z)=0$ follows just as the case $p \neq 2$.

Thus the proof of Theorem is completed.

Remark. In Theorem, if we replace $p^{r}$ by a number which is not a power of an odd prime number, or by $2^{r}(r \geqq 2, \sqrt{-1} \notin K)$ then our Theorem not always holds.

To show this, we use the following well known theorem ([1] p. 198). Let $K$ be a finite algebraic number field and let $M / K$ be a finite Galois extension with Galois group $G=G(M / K)$. For each prime divisor $\mathfrak{p}$ of $K$, we fix a prime divisor $\mathfrak{B}$ of $M$ lying above $\mathfrak{p}$ and let $G_{\mathfrak{P}}$ be the decomposition group of $\mathfrak{P}$. Let $F$ be the subgroup of $H^{-3}(G, Z)$ generated by all $\operatorname{cor}\left(H^{-3}\left(G_{\mathfrak{B}}, Z\right)\right)$ where $\mathfrak{p}$ runs over all prime divisors of $K$ and cor is the correstriction homomorphism from $H^{-3}\left(G_{\mathfrak{B}}, Z\right)$ into $H^{-3}(G, Z)$. Then the theorem asserts that $\operatorname{Knot}(M / K) \approx H^{-3}(G, Z) / F$. 
In the following Examples, $\zeta_{t}$ is a primitive $t$-th root of unity.

EXAMPLE 1. Let $L=\boldsymbol{Q}(\zeta), \zeta=\zeta_{21}$ and let $K$ be the subfield of $L$ which corresponds to the subgroup $\left\langle\tau_{0}\right\rangle$ of $\operatorname{Gal}(L / \boldsymbol{Q})$, where $\tau_{0}(\zeta)=\zeta^{4}$. Then we have $\operatorname{Knot}(M / K) \approx Z / 3 Z$ where $M=L\left(883^{1 / 21}\right)$.

Proof. 883 is a prime number and $883 \equiv 1 \bmod (21)^{2}$. We have $\operatorname{Gal}(M / K)=$ $\langle\sigma, \tau\rangle\left(\sigma(\theta)=\theta \zeta, \sigma(\zeta)=\zeta ; \tau(\theta)=\theta\right.$ and $\tau(\zeta)=\zeta^{4}$ where $\left.\theta=883^{1 / 21}\right), \sigma^{21}=\tau^{3}=1$ and $\tau \sigma \tau^{-1}=\sigma^{4}$. By Lemma, we have $H^{-3}(G, Z) \approx Z / 3 Z$. On the other hand, for any prime divisor $\mathfrak{P}$ of $M$ the decomposition group $G_{\mathfrak{B}}$ is cyclic. For the proof, we may consider only $\mathfrak{P}$ which is above 883,3 or 7 . When $\mathfrak{P}$ is above $883, G_{\mathfrak{F}} \subseteq$ $\operatorname{Gal}(M / L)=\langle\sigma\rangle$ because the prime of $K$ under $\mathfrak{P}$ splits completely in $L$. When $\mathfrak{P}$ is above 3 or 7 the prime of $L$ under $\mathfrak{P}$ splits completely in $M$, because $X^{21} \equiv$ $883 \bmod 3^{2}$ or $\bmod 7^{2}$ has a solution $X=1$. Hence the order of $G_{\mathfrak{P}}$ is $\leqq 3$ and $G_{\Re}$ is cyclic. Therefore for any $\mathfrak{P}, H^{-3}\left(G_{\mathfrak{F}}, Z\right)=0$ and by the above theorem we have $\operatorname{Knot}(M / K) \approx Z / 3 Z$.

EXAMPLE 2. Let $K=\boldsymbol{Q}, L=\boldsymbol{Q}\left(\zeta_{4}\right)=\boldsymbol{Q}(\sqrt{-1})$, and $M=L\left(17^{1 / 4}\right)$, then $\operatorname{Knot}(M / K)$ $\approx Z / 2 Z$.

Proof. $\operatorname{Gal}(M / K)=\langle\sigma, \tau\rangle, \sigma^{4}=\tau^{2}=1$ and $\tau \sigma \tau^{-1}=\sigma^{3}$. By Lemma, we have $H^{3}(G, Z) \approx Z / 2 Z$. On the other hand, just as Example 1 , we see that for any prime divisor $\mathfrak{P}$ of $M, G_{\mathfrak{P}}$ is cyclic and $\operatorname{Knot}(M / K) \approx Z / 2 Z$.

Remark. As we have seen in the proof of Theorem, we have a slightly generalized theorem as follows; let $p$ be an odd prime number and let $M / K$ be a finite Galois extension. If $\operatorname{Gal}(M / K)=\langle\sigma, \tau\rangle, \sigma^{p^{r}}=\tau^{n}=1\left(n \mid \varphi\left(p^{r}\right)\right),\langle\sigma\rangle \cap\langle\tau\rangle=1$, $\tau \sigma \tau^{-1}=\sigma^{m}$ and $m \bmod p^{r}$ has order $n$ in the unit group of $Z / p^{r} Z$, then Knot $(M / K)=1$. We have also a similar generalization for $p=2$.

\section{A Proof of Lemma.}

Let $G$ be a group of the type in Lemma: $G$ is a group of order $l n$, generated by two elements $\sigma, \tau$ with fundamental relations $\sigma^{l}=\tau^{n}=1, \tau \sigma \tau^{-1}=\sigma^{m}$ where $1 \leqq m<l$ and $m^{n}-1$ is a multipler of $l$. In the following, let $N=1+\sigma+\cdots+\sigma^{l-1}$, $\Delta=1-\sigma, \quad S=1+\sigma+\cdots+\sigma^{m-1}, T_{\imath}=\tau^{-1} S^{\imath}, N_{\imath}=1+T_{i}+\cdots+T_{\imath}^{n-1}, \Delta_{\imath}=1-T_{\imath}$ and $L_{\imath}=\frac{\left(l_{0} N+1\right)^{2}-1}{N}$, where $i \geqq 0$ and $l_{0}=\left(m^{n}-1\right) / l$.

For a left $G$-module $A$, in [4], by giving a free resolution of $G$, we determined cohomology groups $H^{r}(G, A)$ as follows:

Proposition. Let $M_{1}=\left(\begin{array}{l}\Delta \\ \Delta_{0}\end{array}\right), M_{2}=\left(\begin{array}{cc}N & 0 \\ \Delta_{1} & -\Delta \\ 0 & N_{0}\end{array}\right)$ and for $q \geqq 1$ 


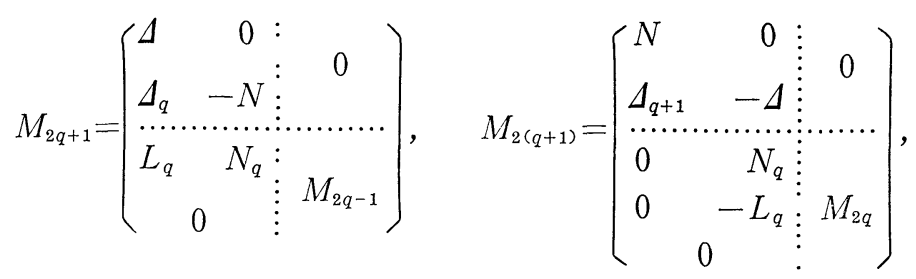

where 0 means that all elements in the places are 0. Then

$$
H^{i}(G, A)=\{a\} /\left\{M_{\imath} b\right\} \quad(i=1,2, \cdots),
$$

where $\{a\}=\left\{a=\left(a_{1}, \cdots, a_{\imath+1}\right)^{t}\right.$ (column vector) $\mid a_{\jmath} \in A$ and $\left.M_{\imath+1} a=0\right\}$ and $\left\{M_{i} b\right\}=$ $\left\{M_{\imath}\left(b_{1}, \cdots, b_{\imath}\right)^{t} \mid b_{\jmath} \in A\right\}$.

Now we prove our Lemma by above Proposition. Since $G$ operates trivially on $Z$, we have, for $r \in Z, N r=l r, \Delta_{2} r=\left(1-m^{2}\right) r, \Delta r=\Delta_{0} r=0, N_{1} r=\mu r \quad(\mu=1+m$ $\left.+\cdots+m^{n-1}\right), L_{1} r=l_{0} r, \Delta_{1} r=(1-m) r$ and $N_{0} r=n r$.

By Proposition, $H^{3}(G, Z) \approx\{a\} /\left\{M_{3} b\right\}$ and direct computations give $\{a\} \approx$ $\left\{x\left(r_{0},-s_{0}\right) \mid x \in Z\right\}$ where $\mu=d_{0} s_{0}, l=d_{0} r_{0}\left(\left(s_{0}, r_{0}=1\right)\right)$ and $\left\{M_{3} b\right\} \approx\{((1-m) y-l z$, $\left.\left.l_{0} y+\mu z\right)^{t} \mid y, z \in Z\right\}=\left\{\left(d_{1} y+d_{0} z\right)\left(r_{0},-s_{0}\right)^{t} \mid y, z \in Z\right\}$, where $d_{1}=\left(m-1, l_{0}\right)$. (For convenience if $m-1=l_{0}=0$ we set $d_{1}=0$.)

Hence $\left\{M_{3} b\right\} \approx\left\{d x\left(r_{0},-s_{0}\right)^{t} \mid x \in Z\right\}$, where $d=\left(d_{0}, d_{1}\right)$. Consequentely we have $H^{3}(G, Z) \approx Z / d Z$, where $d=\left(1+m+\cdots+m^{n-1}, l,\left(m^{n}-1\right) / l, m-1\right)$.

\section{REFERENCES}

[1] J.W.S. Cassels and A. Frohlich, Algebraic Number Theory, Proceedings of a Conference (Brighton 1965), LONDON-NEW YORK ACADEMIC PRESS 1967.

[2] D.A. Garbanti, The Hasse norm theorem for $l$-extensions of the rationals, In: Number and Algebra (ed. H. ZASSENHAUS), LONDON-NEW YORK ACADEMIC PRESS (1977), 77-90.

[3] D.A. Garbanti, The Hasse norm theorem for non-cyclic extensions of the rationals, Proc. London Math. Soc. (3) 37 (1978), 143-164.

[4] S. Hamada, On cohomology groups of dihedral groups (in Japanese), "Sūgaku" Vol. 16 (2) (1964), 106-107.

[5] H. HASSE, Beweis eines Satzes und Widerlegung einer Vermutung über das allgemeine Normenrestsymbol, Nach. Ges. Wiss. 1 (1931), 64-69.

[6] F. Lolenz, Über eine Verallgemeierung des Hasseschen Normensatzes, Math. $Z$. 173 (1980), 203-210.

[ ] H. Opolka, Zur Auflösung zahlentheoretıscher Knoten, Math. Z. 173 (1980), 95103.

Department of Mathematics

MiYagi University of Education

SENDAI 